\title{
Some notes on Teichmüller shift mappings and the Teichmüller density
}

\author{
By \\ SHEN Yu-Liang*
}

\begin{abstract}
Kra $[\mathrm{Kr}]$ introduced a distance $d_{K}$ on every hyperbolic Riemann surface $R$ by means of Teichmüller shift mappings. Recently Gardiner and Lakic [GL2] defined a metric density $\lambda$, the Teichmüller density, on such a surface. The paper deals with some basic properties of the Teichmüller density $\lambda$ and the distance $d_{K}$, giving some close relation between them. Particularly, it is shown that the distance function $d_{K}: R \times R \rightarrow \mathbb{R}$ is continuously differentiable off the diagonal, and the Teichmüller density $\lambda$ is precisely the metric density of the infinitesimal form of the distance $d_{K}$ and it is continuous on the whole surface $R$. Some related topics will also be discussed.
\end{abstract}

\section{Introduction}

When studying the self-maps of Riemann surfaces and the geometry of Teichmüller spaces, Kra $[\mathrm{Kr}]$ introduced a distance $d_{K}$ on every hyperbolic Riemann surface $R$ by means of Teichmüller shift mappings. It is known that the distance $d_{K}$ has some close relation with the hyperbolic distance on $R$ (see [EKK], [EL1], [EL2], [Ge], [Kr], [Kru3], [Liu], [Na1], [Re], [St2], [Te]). Recently, by using the infinitesimal Teichmüller norms of certain vector fields to Teichmüller spaces, Gardiner and Lakic [GL2] defined a metric density $\lambda$, the Teichmüller density, on such a surface $R$. They also use the Teichmüller density $\lambda$ to study the hyperbolic density on $R$ and characterize the uniform perfectness of closed sets in the complex plane $\overline{\mathbb{C}}$. In this note, we shall give some basic properties of the Teichmüller density $\lambda$ and the distance $d_{K}$, giving some close relation between them. Particularly, we shall show that the Teichmüller density $\lambda$ is precisely the metric density of the infinitesimal form of the distance $d_{K}$ (see Theorems 1 and 2), that the distance function $d_{K}: R \times R \rightarrow \mathbb{R}$

1991 Mathematics Subject Classification(s). 30F60, 32G15, 30C62, 30C70

Key words: Teichmüller density, hyperbolic density, Teichmüller shift mapping

Received March 18, 2002

Revised July 29, 2002

*Project supported by the National Natural Science Foundation of China and the Natural Science Foundation of Jiangsu Provincial Education Committee. 
is continuously differentiable off the diagonal (see Theorem 6), and that the Teichmüller density $\lambda$ is continuous on the whole surface $R$ (see Theorem 4). We shall prove these results by studying the Teichmüller shift mappings and some holomorphic mappings from Riemann surfaces into Teichmüller spaces, and as by-products, we shall prove some results on the continuity of point shift differentials and extremal differentials (see Theorems 3 and 5).

\section{Preliminaries}

In this section, we shall give the precise definitions of Kra's distance $d_{K}$ and the Teichmüller density $\lambda$. As already stated in Section 1, they are defined respectively by using the Teichmüller shift mappings and the infinitesimal Teichmüller norms of certain vector fields to Teichmüller spaces, and have close relation with the hyperbolic distance and the hyperbolic density, so we need to recall some basic definitions and fundamental results from hyperbolic geometry and quasiconformal Teichmüller theory.

We begin with the basic definitions and notations on hyperbolic density (and distance). Let $\Delta=\{z:|z|<1\}$ denote the unit disk on the complex plane $\overline{\mathbb{C}}$. On $\Delta$ one can define the hyperbolic Poincaré metric $G_{H, \Delta}: T \Delta=$ $\Delta \times \mathbb{C} \rightarrow \mathbb{R}$ by

$$
G_{H, \Delta}(z, v)=\frac{|v|}{1-|z|^{2}}
$$

with density $\rho_{\Delta}=\rho_{\Delta}(z)|d z|$ by

$$
\rho_{\Delta}(z)=\frac{G_{H, \Delta}(z, v)}{|v|}=\frac{1}{1-|z|^{2}} .
$$

The Poincaré distance $d_{H, \Delta}\left(z_{1}, z_{2}\right)$ between two points $z_{1}, z_{2}$ induced by $\rho_{\Delta}$ is

$$
d_{H, \Delta}\left(z_{1}, z_{2}\right)=\frac{1}{2} \log \frac{1+\left|\frac{z_{1}-z_{2}}{1-\overline{z_{1}} z_{2}}\right|}{1-\left|\frac{z_{1}-z_{2}}{1-\overline{z_{1}} z_{2}}\right|} .
$$

Let $R$ be a hyperbolic Riemann surface covered by the unit disk $\Delta$. Then there is a Fuchsian group $\Gamma$ such that $R=\Delta / \Gamma$. Let $\pi: \Delta \rightarrow R$ denote the canonical holomorphic projection. On $R$ one can define the hyperbolic metric $G_{H, R}: T R \rightarrow \mathbb{R}$ by requiring that $\pi^{*} G_{H, R}=G_{H, \Delta}$, or precisely, for any $(z, v) \in T \Delta$, that

$$
G_{H, R}\left(\pi(z), d_{z} \pi(v)\right)=G_{H, \Delta}(z, v),
$$

with density $\rho_{R}=\rho_{R}(w)|d w|$ by

$$
\rho_{R}(w(\pi(z)))\left|(w \circ \pi)^{\prime}(z)\right|=\rho_{\Delta}(z)=\frac{1}{1-|z|^{2}},
$$


where $w$ is any local parameter on $R$. The hyperbolic distance $d_{H, R}\left(p_{1}, p_{2}\right)$ between two points $p_{1}, p_{2}$ induced by $\rho_{R}$ is

$$
d_{H, R}\left(p_{1}, p_{2}\right)=\inf \left\{d_{H, \Delta}\left(z_{1}, z_{2}\right): \pi\left(z_{1}\right)=p_{1}, \pi\left(z_{2}\right)=p_{2}\right\} .
$$

In the following, when there is no ambiguity, we set $d_{H, R}=d_{H}, G_{H, R}=G_{H}$, $\rho_{R}=\rho$.

Now we begin to define Kra's distance $d_{K}$ by Teichmüller shift mappings. A (classical) Teichmüller shift mapping (see [Te]) is the uniquely extremal mapping $T_{\delta}$ which sends the zero point to $-\delta(0 \leq \delta<1)$ and fixes every boundary point of the unit disk $\Delta$. It is a Teichmüller mapping with Beltrami coefficient $\mu_{\delta}$ such that $\mu_{0}=0$, while for $\delta>0, \mu_{\delta}=k_{\delta}\left|\phi_{\delta}\right| / \phi_{\delta}$, where $k_{\delta}>0$, and $\phi_{\delta}$ is a holomorphic function in $\Delta-\{0\}$, which has a first order pole at 0 and has unit $L^{1}$-norm.

In general, a Teichmüller shift mapping on a hyperbolic Riemann surface $R$ is the uniquely extremal mapping $T_{p_{1}, p_{2}}$ which sends $p_{1}$ to $p_{2}$ and is homotopic to the identity mapping modulo the ideal boundary $\partial R$. It is a Teichmüller mapping with Beltrami coefficient $\mu_{p_{1}, p_{2}}$ such that, for $p_{2}=p_{1}, \mu_{p_{1}, p_{2}}=0$, while for $p_{2} \neq p_{1}, \mu_{p_{1}, p_{2}}=k_{p_{1}, p_{2}}\left|\phi_{p_{1}, p_{2}}\right| / \phi_{p_{1}, p_{2}}$, where $k_{p_{1}, p_{2}}>0$, and $\phi_{p_{1}, p_{2}}$ is a holomorphic quadratic differential in $R-\left\{p_{1}\right\}$, which has a first order pole at $p_{1}$ and has unit $L^{1}$-norm. Following [St2], we call $\phi_{p_{1}, p_{2}}$ a point shift differential.

It is known that the Teichmüller shift mapping plays an important role in the theory of extremal quasiconformal mappings and Teichmüller spaces (see [EL2], [Ge], [Kr], [Re], [St2]) and in classical complex analysis (see [Kru3]) as well. As stated in the beginning, when studying the self-maps of Riemann surfaces and the geometry of Teichmüller spaces, Kra [Kr] introduced a distance $d_{K}$ on every hyperbolic Riemann surface $R$ by the Teichmüller shift mappings. The precise definition is as follows:

Definition 1. For any two points $p_{1}$ and $p_{2}$ in a surface $S$, Kra's distance $d_{K}$ is defined as

$$
d_{K}\left(p_{1}, p_{2}\right)=\frac{1}{2} \log \frac{1+k_{p_{1}, p_{2}}}{1-k_{p_{1}, p_{2}}} .
$$

We have the following known results.

Theorem A. The identity mapping $i d:\left(R, d_{H}\right) \rightarrow\left(R, d_{K}\right)$ is not an isometry unless $R=\overline{\mathbb{C}}_{p q r}=\overline{\mathbb{C}}-\{p, q, r\}$.

Remark 1. When $R$ is of conformally finite type, Theorem A was proved independently by Kra [Kr] and Nag [Na1]. Later Liu [Liu] extended it to all hyperbolic Riemann surfaces of conformally infinite type with three exceptions: the cases when $R$ is $\Delta, \Delta$ with one puncture, or an annulus. Very recently, Earle and Lakic [EL2] gave an explicit and elementary proof of Theorem $\mathrm{A}$ in all cases. 
Remark 2. It is a classical result of Teichmüller (see [Li]) that when $R$ is the punctured sphere $\overline{\mathbb{C}}_{p q r}, d_{K}=d_{H}$. On the other hand, when $R$ is simply connected, it is easy to see that $d_{K}$ is uniquely determined by $d_{H}$. In fact, we have the following exact formula:

$$
\log \frac{e^{d_{K}}+1}{e^{d_{K}}-1}=\mu\left(\frac{e^{2 d_{H}}-1}{e^{2 d_{H}}+1}\right),
$$

where $\mu(r)$ is the conformal module of the Grötzsch ring domain whose boundary components are the unit circle and the line segment $\{x: 0 \leq x \leq r\}$. Noting that $\mu(r)=(1+o(1)) \log (4 / r)$ as $r \rightarrow 0$, we find that $d_{K}=d_{H} / 2+o\left(d_{H}\right)$ as $d_{H} \rightarrow 0$. For more details, see the papers [Re], [Te].

It is well known that the hyperbolic metric $G_{H}$ is the infinitesimal form of the hyperbolic distance $d_{H}$, namely, for any $z \in \Delta$ and any number $v \in \mathbb{C}$, it holds that

$$
G_{H}\left(\pi(z), d_{z} \pi(v)\right)=\lim _{t \rightarrow 0+} \frac{d_{H}(\pi(z), \pi(z+t v))}{t} .
$$

Now we let $G_{K}$ denote the infinitesimal form of the distance $d_{K}$, that is, for any $z \in \Delta$ and any number $v \in \mathbb{C}$, we have

$$
G_{K}\left(\pi(z), d_{z} \pi(v)\right)=\lim _{t \rightarrow 0+} \frac{d_{K}(\pi(z), \pi(z+t v))}{t} .
$$

Then, as stated in [EL2], a consequence of Theorems A and 5 of [EKL] is

Corollary B. If $R$ is not the punctured sphere $\overline{\mathbb{C}}_{p q r}$, then $d_{K}\left(p_{1}, p_{2}\right)<$ $d_{H}\left(p_{1}, p_{2}\right)$ for any pair of distinct points $p_{1}, p_{2}$ in $R$. In addition, $G_{K}<G_{H}$ except at the points on the zero section.

Remark 3. As remarked above, when $R$ is the punctured sphere $\overline{\mathbb{C}}_{p q r}$, $G_{K}=G_{H}$, when $R$ is simply connected, $G_{K}=G_{H} / 2$. We shall show that $G_{K}>G_{H} / 2$ whenever $R$ is not simply connected, except at the points on the zero section.

Finally, we recall some basic definitions and some fundamental results from the theory of extremal quasiconformal mappings and Teichmüller spaces and define the Teichmüller density $\lambda$. For more details, see the papers [Ha], [Kru1], [RS2], [St1] and the books [Ga], [GL1].

Let $M(S)$ denote the unit ball of the space $\operatorname{Belt}(S)$ of all essentially bounded Beltrami differentials $\mu=\mu(w) d \bar{w} / d w$ on a hyperbolic surface $S$, and $S A(S)$ the unit sphere of the space $A(S)$ of all holomorphic quadratic differentials $\phi=\phi(w) d w^{2}$ on $S$ with finite $L^{1}$-norm

$$
\|\phi\|=\iint_{S}|\phi|<+\infty
$$

For a given $\mu \in M(S)$, denote by $f^{\mu}$ the uniquely determined quasiconformal mapping on $S$ with Beltrami coefficient $\mu$ and some normalized condition which 
can be specified from context. Two elements $\mu$ and $\nu$ in $M(S)$ are equivalent, and denoted by $\mu \sim \nu$, if $f^{\mu}$ is homotopic to $f^{\nu}(\bmod \partial S)$. Then $T(S)=$ $M(S) / \sim$ is the Teichmüller space of $S$. Let $\Phi$ denote the canonical holomorphic projection $M(S) \rightarrow T(S)$, and $d_{\mu} \Phi$ denote the differential of $\Phi$ at the point $\Phi(\mu)$. We also denote by $M(\mu, S)$ the set of all elements $\nu \in M(S)$ equivalent to $\mu$, and set

$$
k(\mu)=\inf \left\{\|\nu\|_{\infty}: \nu \in M(\mu, S)\right\} .
$$

We say that $\nu \in M(\mu, S)$ is extremal if $\|\nu\|_{\infty}=k(\mu)$. It is known that there always exists at least one extremal element in the class $M(\mu, S)$. A quasiconformal mapping $f$ on $S$ is said to be extremal if its Beltrami coefficient $\mu$ is extremal in its own class $M(\mu, S)$. The Teichmüller distance between two points $\Phi\left(\mu_{1}\right)$ and $\Phi\left(\mu_{2}\right)$ in $T(S)$ is defined as

$$
d_{T}\left(\Phi\left(\mu_{1}\right), \Phi\left(\mu_{2}\right)\right)=\frac{1}{2} \log \frac{1+k(\mu)}{1-k(\mu)},
$$

where $\mu$ is the Beltrami coefficient of the mapping $f^{\mu_{2}} \circ\left(f^{\mu_{1}}\right)^{-1}$.

Two elements $\mu$ and $\nu$ in $\operatorname{Belt}(S)$ are infinitesimally equivalent and denoted by $\mu \approx \nu$, if $\iint_{S} \mu \phi=\iint_{S} \nu \phi$ for all $\phi \in A(S)$. Then $\mu \approx \nu$ iff $d_{0} \Phi(\mu)=d_{0} \Phi(\nu)$. So $B(S)=\operatorname{Belt}(S) / \approx$ is the tangent space of $T(S)$ at the base point $\Phi(0)$. We denote by $\operatorname{Belt}(\mu, S)$ the set of all elements $\nu$ in $\operatorname{Belt}(S)$ infinitesimally equivalent to $\mu$ and set

$$
\|\mu\|_{S}=\inf \left\{\|\nu\|_{\infty}: \nu \in \operatorname{Belt}(\mu, S)\right\} .
$$

By the Hahn-Banach extension theorem and Riesz representative theorem from functional analysis theory, $\|\mu\|_{S}$ has another equivalent definition, namely,

$$
\|\mu\|_{S}=\sup _{\phi \in S A(S)}\left|\iint_{S} \mu \phi\right| .
$$

We say that $\nu \in \operatorname{Belt}(\mu, S)$ is infinitesimally extremal if $\|\nu\|_{\infty}=\|\mu\|_{S}$. Again, there always exists at least one infinitesimally extremal element in the class $\operatorname{Belt}(\mu, S)$.

Now we can define the Teichmüller density $\lambda=\lambda(w)|d w|$ introduced by Gardiner and Lakic [GL2] as follows:

Definition 2. For any point $p \in R$, choose some local parameter $w$ on some neighborhood of $p$ with $w(p)=w_{0}$. Choose some vector field $V(w)(\partial / \partial w)$ on $R$ such that $V\left(w_{0}\right)=1, V(\phi)=: \iint_{R} \bar{\partial} V \phi=0$ for all $\phi \in A(R)$, that is, $\bar{\partial} V \in \operatorname{Belt}(0, R)$. Then, with $R_{p}=R-\{p\}$,

$$
\lambda\left(w_{0}\right)=:\|\bar{\partial} V\|_{R_{p}}=\sup _{\phi \in S A\left(R_{p}\right)}\left|\iint_{R} \bar{\partial} V \phi\right| .
$$


We proceed to state some basic results on extremality and infinitesimal extremality of Beltrami differentials. Hamilton, Krushkal, Reich and Strebel showed that a Beltrami coefficient $\mu \in M(S)$ is extremal if and only if it is infinitesimally extremal, which can happen precisely when $\mu$ satisfies the Hamilton-Krushkal condition $\|\mu\|_{\infty}=\|\mu\|_{S}$. In this case, there exists a socalled Hamilton sequence $\left(\phi_{n}\right)$ in $S A(S)$ such that $\iint_{S} \mu \phi_{n} \rightarrow\|\mu\|_{\infty}$ as $n \rightarrow \infty$. Furthermore, either $\mu$ is Teichmüller, meaning as usual that $\mu=\|\mu\|_{\infty}|\phi| / \phi$ for some $\phi \in S A(S)$, or $\left(\phi_{n}\right)$ is degenerating in the sense that $\phi_{n} \rightarrow 0$ locally uniformly in $S$.

Now let $\mu \in M(S)$ and set

$$
h(\mu)=\inf \left\{\left\|\left.\nu\right|_{S-E}\right\|_{\infty}: \nu \in M(\mu, S), E \subset S \text { compact }\right\} .
$$

Clearly, $h(\mu) \leq k(\mu) \leq\|\mu\|_{\infty}$. If $h(\mu)<k(\mu)$, then, by the Frame Mapping Theorem, $M(\mu, S)$ contains a Teichmüller extremal $k(\mu)|\phi| / \phi$, and any Hamilton sequence for $k(\mu)|\phi| / \phi$ must converge in norm to $\phi$. In this case, following [ELi], we call $\Phi(\mu)$ a Strebel point.

Let $\mu \in \operatorname{Belt}(S)$. Following Earle-Gardiner [EG], let

$$
\beta(\mu)=\sup \limsup _{n \rightarrow \infty}\left|\iint_{S} \mu \phi_{n}\right|,
$$

where the supremum is taken over all degenerating sequences $\left(\phi_{n}\right)$ in $S A(S)$. Clearly, $\beta(\mu) \leq\|\mu\|_{S} \leq\|\mu\|_{\infty}$. If $\beta(\mu)<\|\mu\|_{S}$, then, by the Infinitesimal Frame Mapping Theorem, Belt $(\mu, S)$ contains a Teichmüller infinitesimal extremal $\|\mu\|_{S}|\phi| / \phi$ and any Hamilton sequence for $\|\mu\|_{S}|\phi| / \phi$ must also converge in norm to $\phi$. In this case, we call $d_{0}(\Phi)(\mu)$ an infinitesimal Reich-Strebel point.

We will make essential use of the following results:

Proposition C. If $M(\mu, S)$ contains a Teichmüller extremal $k(\mu)|\phi| / \phi$, then

$$
\frac{1+k(\mu)}{1-k(\mu)} \leq \iint_{S}|\phi| \frac{\left|1+\mu \frac{\phi}{|\phi|}\right|^{2}}{1-|\mu|^{2}} .
$$

Proposition D. For any Beltrami coefficient $\mu \in \operatorname{Belt}(S)$, if $\mu_{t}=t \mu+$ $o(t)$ uniformly in $S$, then

$$
k\left(\mu_{t}\right)=|t|\|\mu\|_{S}+o(t)=|t| \sup _{\phi \in S A(S)}\left|\iint_{S} \mu \phi\right|+o(t) .
$$

From (2.18) one can deduce that the infinitesimal form $G_{T}: T T(S) \rightarrow \mathbb{R}$ of the Teichmüller distance $d_{T}$ is

$$
\begin{aligned}
G_{T}\left(\Phi(\mu), d_{\mu} \Phi(\nu)\right) & =\inf \left\{\left\|\frac{\tilde{\nu}}{1-|\mu|^{2}}\right\|_{\infty}: d_{\mu} \Phi(\tilde{\nu})=d_{\mu} \Phi(\nu)\right\} \\
& =\sup _{\phi \in S A\left(f^{\mu}(S)\right)}\left|\iint_{f^{\mu}(S)}\left(\frac{\nu}{1-|\mu|^{2}} \frac{\partial f^{\mu}}{\partial f^{\mu}}\right) \circ\left(f^{\mu}\right)^{-1} \phi\right| .
\end{aligned}
$$




\section{The infinitesimal form $G_{K}$ and the Teichmüller density $\lambda$}

In this section, we shall show that the Teichmüller density $\lambda$ is precisely the metric density of the infinitesimal form $G_{K}$. We first give some expressions of the infinitesimal form $G_{K}$ and give a lower bound of $G_{K}$ as a supplement to Corollary B.

Recall that $p=\pi(\zeta)$ is the universal covering from $\Delta$ onto $R$. In the following we shall use $\zeta=\pi^{-1}$ as a local parameter. Let $p \in R$ be given. Choose $z_{0} \in \Delta$ with $\pi\left(z_{0}\right)=p$. Then there exists some $r>0$ such that $\pi$ is injective in $\Delta_{r}=\left\{\zeta:\left|\zeta-z_{0}\right|<r\right\}$. Set $D_{r}=\pi\left(\Delta_{r}\right)$. Then for any $q \in D_{r}$ there exists a unique $z \in \Delta_{r}$ with $\pi(z)=q$. Now we define

$$
\tilde{f}(\zeta)=\zeta+\frac{z-z_{0}}{r}\left(r-\left|\zeta-z_{0}\right|\right), \quad \zeta \in \Delta_{r},
$$

and set

$$
f=\chi_{D_{r}} \pi \circ \tilde{f} \circ \pi^{-1}+\chi_{R-D_{r}} i d,
$$

where $\chi$ denotes the characteristic function of a set. Clearly, $f$ is homotopic to the Teichmüller shift mapping $T_{p, q}$ modulo $\partial R_{p}$.

Now a direct computation will show that the Beltrami coefficient of $\tilde{f}$ is

$$
\tilde{\mu}(\zeta)=\frac{\left(z-z_{0}\right)\left(\zeta-z_{0}\right)}{\left(z-z_{0}\right) \overline{\left(\zeta-z_{0}\right)}-2 r\left|\zeta-z_{0}\right|}=-\frac{z-z_{0}}{2 r} \frac{\zeta-z_{0}}{\left|\zeta-z_{0}\right|}+o\left(\left|z-z_{0}\right|\right) .
$$

By definition, the Beltrami coefficient of $f$ is $\mu=\chi_{\Delta_{r}} \tilde{\mu}$. So we get from (3.3) that

$$
\mu(\zeta)=-\frac{z-z_{0}}{2 r} \frac{\zeta-z_{0}}{\left|\zeta-z_{0}\right|} \chi_{\Delta_{r}}+o\left(\left|z-z_{0}\right|\right) .
$$

Now we can apply Proposition D to $\mu$ and obtain

$$
k_{p, q}=\frac{\left|z-z_{0}\right|}{2 r} \sup _{\phi \in S A\left(R_{p}\right)}\left|\iint_{\Delta_{r}} \frac{\zeta-z_{0}}{\left|\zeta-z_{0}\right|} \phi\right|+o\left(\left|z-z_{0}\right|\right) .
$$

Noting that $d_{H}(p, q)=d_{H}\left(z_{0}, z\right)$, we conclude that

$$
\left|z-z_{0}\right|=\left(1-\left|z_{0}\right|^{2}\right) d_{H}(p, q)+o\left(d_{H}(p, q)\right) .
$$

Consequently,

$$
k_{p, q}=\frac{\left(1-\left|z_{0}\right|^{2}\right) d_{H}(p, q)}{2 r} \sup _{\phi \in S A\left(R_{p}\right)}\left|\iint_{\Delta_{r}} \frac{\zeta-z_{0}}{\left|\zeta-z_{0}\right|} \phi\right|+o\left(d_{H}(p, q)\right) .
$$

So

$$
\begin{aligned}
d_{K}(p, q) & =\frac{1}{2} \log \frac{1+k_{p, q}}{1-k_{p, q}}=k_{p, q}+o\left(k_{p, q}\right) \\
& =\frac{\left(1-\left|z_{0}\right|^{2}\right) d_{H}(p, q)}{2 r} \sup _{\phi \in S A\left(R_{p}\right)}\left|\iint_{\Delta_{r}} \frac{\zeta-z_{0}}{\left|\zeta-z_{0}\right|} \phi\right|+o\left(d_{H}(p, q)\right) .
\end{aligned}
$$


Thus

$$
\lim _{q \rightarrow p} \frac{d_{K}(p, q)}{d_{H}(p, q)}=\frac{\left(1-\left|z_{0}\right|^{2}\right)}{2 r} \sup _{\phi \in S A\left(R_{p}\right)}\left|\iint_{\Delta_{r}} \frac{\zeta-z_{0}}{\left|\zeta-z_{0}\right|} \phi\right| .
$$

Particularly,

$$
G_{K}\left(p, v_{p}\right)=\frac{\left(1-\left|z_{0}\right|^{2}\right) G_{H}\left(p, v_{p}\right)}{2 r} \sup _{\phi \in S A\left(R_{p}\right)}\left|\iint_{\Delta_{r}} \frac{\zeta-z_{0}}{\left|\zeta-z_{0}\right|} \phi\right| .
$$

Now

$$
\iint_{\Delta_{r}} \frac{\zeta-z_{0}}{\left|\zeta-z_{0}\right|} \phi=\int_{0}^{r} s d s \int_{|\zeta|=s} \frac{\zeta}{s} \phi\left(\zeta+z_{0}\right) \frac{d \zeta}{i \zeta}=2 \pi r \operatorname{Res}_{\zeta=z_{0}} \phi(\zeta),
$$

where and in what follows, $\operatorname{Res}_{\zeta=z_{0}} \phi(\zeta)$ denotes the residue of $\phi(\zeta)$ at $z_{0}$. It should be noted that the residue $\operatorname{Res}_{\zeta=z_{0}} \phi(\zeta)$ depends on the choice of the local parameter $z_{0}$. So

$$
G_{K}\left(p, v_{p}\right)=\pi\left(1-\left|z_{0}\right|^{2}\right) G_{H}\left(p, v_{p}\right) \sup _{\phi \in S A\left(R_{p}\right)}\left|\operatorname{Res}_{\zeta=z_{0}} \phi(\zeta)\right| .
$$

To get a lower bound of $G_{K}\left(p, v_{p}\right)$, as done in [GL2], we use the Poincaré theta series operator $\Theta_{\Gamma}$ (see $\left.[\mathrm{Ga}]\right)$, which is defined as

$$
\Theta_{\Gamma} \psi=\sum_{\gamma \in \Gamma} \psi \circ \gamma\left(\gamma^{\prime}\right)^{2}
$$

Letting

$$
\psi(\zeta)=\frac{\left(1-\left|z_{0}\right|^{2}\right)^{2}}{\left(\zeta-z_{0}\right)\left(1-\overline{z_{0}} \zeta\right)^{3}}
$$

and $\phi=\Theta_{\Gamma} \psi$. Then $\phi=\phi(\zeta) d \zeta^{2} \in A\left(R_{p}\right)$, and $\|\phi\|=\left\|\Theta_{\Gamma} \psi\right\| \leq\|\psi\|=2 \pi$. Now

$$
\operatorname{Res}_{\zeta=z_{0}} \phi(\zeta)=\frac{1}{1-\left|z_{0}\right|^{2}} .
$$

So $G_{K}\left(p, v_{p}\right) \geq G_{H}\left(p, v_{p}\right) / 2$. Note that if the equality holds for a non-zero vector $v_{p}$, then $\|\phi\|=\left\|\Theta_{\Gamma} \psi\right\|=\|\psi\|=2 \pi$, which implies that $\Gamma$ is the trivial group, or equivalently, $R$ is simply connected. As remarked in Section 2, the converse is also true.

We can summarize the above as

Theorem 1. The infinitesimal form $G_{K}$ of the distance $d_{K}$ has the expression (3.10) and (3.12). When $R$ is the punctured sphere $\overline{\mathbb{C}}_{p q r}, G_{K}=G_{H}$, when $R$ is simply connected, $G_{K}=G_{H} / 2$, in all other cases, $G_{H} / 2<G_{K}<$ $G_{H}$ except at the points on the zero section.

Now we show that the Teichmüller density $\lambda$ is precisely the metric density of the infinitesimal form $G_{K}$. Let

$$
V(\zeta)=\left(1-\frac{\left|\zeta-z_{0}\right|}{r}\right) \chi_{\Delta_{r}} .
$$


Clearly, $V\left(z_{0}\right)=1$. For any $\phi \in A\left(R_{p}\right)$, noting that

$$
\bar{\partial} V=-\frac{1}{2 r} \frac{\zeta-z_{0}}{\left|\zeta-z_{0}\right|} \chi_{\Delta_{r}}
$$

we obtain from (3.11) that

$$
V(\phi)=\iint_{R} \bar{\partial} V \phi=-\frac{1}{2 r} \iint_{\Delta_{r}} \frac{\zeta-z_{0}}{\left|\zeta-z_{0}\right|} \phi=-\pi \operatorname{Res}_{\zeta=z_{0}} \phi .
$$

So $V(\phi)=0$ for all $\phi \in A(R)$, and

$$
\lambda\left(z_{0}\right)=\frac{1}{2 r} \sup _{\phi \in S A\left(R_{p}\right)}\left|\iint_{\Delta_{r}} \frac{\zeta-z_{0}}{\left|\zeta-z_{0}\right|} \phi\right|=\pi \sup _{\phi \in S A\left(R_{p}\right)}\left|\operatorname{Res}_{\zeta=z_{0}} \phi\right| .
$$

By (3.12) and (3.15), noting that $\rho\left(z_{0}\right)=1 /\left(1-\left|z_{0}\right|^{2}\right)$, we obtain

$$
\frac{G_{K}\left(p, v_{p}\right)}{G_{H}\left(p, v_{p}\right)}=\frac{\lambda\left(z_{0}\right)}{\rho\left(z_{0}\right)}
$$

which implies that $\lambda$ is the metric density of $G_{K}$. We have obtained

Theorem 2. The Teichmüller density $\lambda$ is the metric density of the infinitesimal form $G_{K}$ and has the expression (3.15). When $R$ is the punctured sphere $\overline{\mathbb{C}}_{p q r}, \lambda=\rho$, when $R$ is simply connected, $\lambda=\rho / 2$, in all other cases, $\rho / 2<\lambda<\rho$.

\section{Point shift differential and extremal differential}

Since $\bar{\partial} V \in \operatorname{Belt}(0, R)$, we conclude that $\beta(\bar{\partial} V)=0$. By the Infinitesimal Frame Mapping Theorem, there exists unique $\phi$ in $S A\left(R_{p}\right)$, which we denote by $\phi_{p}$, such that $\lambda\left(z_{0}\right)\left|\phi_{p}\right| / \phi_{p} \in \operatorname{Belt}\left(\bar{\partial} V, R_{p}\right)$. For simplicity, we shall call $\phi_{p}$ an extremal differential (at the point $p$ ). Note that by definition,

$$
\begin{aligned}
\lambda\left(z_{0}\right) & =\sup _{\phi \in S A\left(R_{p}\right)} \operatorname{Re} \iint_{R} \bar{\partial} V \phi \\
& =\frac{1}{2 r} \sup _{\phi \in S A\left(R_{p}\right)} \operatorname{Re} \iint_{\Delta_{r}} \frac{\zeta-z_{0}}{\left|\zeta-z_{0}\right|} \phi \\
& =\pi \sup _{\phi \in S A\left(R_{p}\right)} \operatorname{Re}\left(\operatorname{Res}_{\zeta=z_{0}} \phi\right) .
\end{aligned}
$$

Then $\phi_{p}$ is the unique element in $S A\left(R_{p}\right)$ which attains the first supremum in (4.1), and every element $\phi \in A\left(R_{p}\right)$ satisfies

$$
\operatorname{Res}_{\zeta=z_{0}} \phi=-\frac{\lambda\left(z_{0}\right)}{\pi} \iint_{R} \frac{\left|\phi_{p}\right|}{\phi_{p}} \phi .
$$

Here it should be pointed out that the extremal differential $\phi_{p}$ depends on the choice of the local parameter $z_{0}$. 
Remark 4. This is a convenient place to point out that the Teichmüller density $\lambda=\lambda_{R}$ is monotone, namely, $\lambda_{R_{0}} \geq \lambda_{R}$ holds for each Riemann surface and for each subdomain $R_{0}$ of $R$. In fact, if $p \in R_{0}$, and $\pi\left(z_{0}\right)=p$, then $\lambda_{R_{0}}\left(z_{0}\right)=\pi \sup _{\psi \in S A\left(R_{0 p}\right)}\left|\operatorname{Res}_{\zeta=z_{0}} \psi\right|, \lambda_{R}\left(z_{0}\right)=\pi \sup _{\phi \in S A\left(R_{p}\right)}\left|\operatorname{Res}_{\zeta=z_{0}} \phi\right|$. Noting that for any $\phi \in S A\left(R_{p}\right), \psi=\phi /\|\phi\|_{R_{0}} \in S A\left(R_{0 p}\right)$, we conclude that $\lambda_{R_{0}}\left(z_{0}\right) \geq \lambda_{R}\left(z_{0}\right)$.

Remark 5. It is known that the Poincaré density is holomorphically contractive, namely, $f^{*} \rho_{S} \leq \rho_{R}$ for any holomorphic mapping $f$ between two Riemann surfaces $R$ and $S$, and it is invariant under covering projections, that is, $f^{*} \rho_{S}=\rho_{R}$ when $f: R \rightarrow S$ is a covering. Neither is true for the Teichmüller density $\lambda$, however. In fact, let $\pi: \Delta \rightarrow R$ be a universal covering for a nonsimply connected Riemann surface $R$, then $\pi^{*} \lambda_{R}>\pi^{*}\left(\rho_{R} / 2\right)=\rho_{\Delta} / 2=\lambda_{\Delta}$, except at the points on the zero section. More generally, for any covering mapping $\pi: R \rightarrow S$ between two Riemann surfaces, it is easy to see that $\pi^{*} \lambda_{S} \geq \lambda_{R}$

We recall that $k_{p, q}\left|\phi_{p, q}\right| / \phi_{p, q}$ is the Beltrami coefficient of the Teichmüller shift mapping $T_{p, q}$. Now we prove

Theorem 3. Under the notations before $\left(\pi\left(z_{0}\right)=p, \pi(z)=q, d_{\rho}\left(z_{0}, z\right)\right.$ $\left.=d_{\rho}(p, q)\right)$,

(1) $\lim _{q \rightarrow p}\left\|\left(z-z_{0}\right) /\left(\left|z-z_{0}\right|\right) \phi_{p, q}-\phi_{p}\right\|=0$;

(2) $\lim _{q \rightarrow p}\left\|\left(z-z_{0}\right) /\left(\left|z-z_{0}\right|\right) \phi_{q, p}+\phi_{p}\right\|=0$.

Proof. We apply Proposition C to $\mu$ (which is the Beltrami coefficient of the mapping $f$ by (3.2)) and $k_{p, q}\left|\phi_{p, q}\right| / \phi_{p, q}$ and obtain

$$
\frac{1+k_{p, q}}{1-k_{p, q}} \leq \iint_{R}\left|\phi_{p, q}\right| \frac{\left|1+\mu \frac{\phi_{p, q}}{\left|\phi_{p, q}\right|}\right|^{2}}{1-|\mu|^{2}} .
$$

By (3.5) and (3.15) we get

$$
k_{p, q}=\left|z-z_{0}\right| \lambda\left(z_{0}\right)+o\left(\left|z-z_{0}\right|\right)
$$

and so

$$
\frac{1+k_{p, q}}{1-k_{p, q}}=1+2\left|z-z_{0}\right| \lambda\left(z_{0}\right)+o\left(\left|z-z_{0}\right|\right) .
$$

By (3.4) and (3.14) we have

$$
\mu(\zeta)=\left(z-z_{0}\right) \bar{\partial} V(\zeta)+o\left(\left|z-z_{0}\right|\right)
$$

and so

$$
\iint_{R}\left|\phi_{p, q}\right| \frac{\left|1+\mu \frac{\phi_{p, q}}{\left|\phi_{p, q}\right|}\right|^{2}}{1-|\mu|^{2}}=1+2 \operatorname{Re} \iint_{R}\left(z-z_{0}\right) \bar{\partial} V \phi_{p, q}+o\left(\left|z-z_{0}\right|\right) .
$$


Since $\lambda\left(z_{0}\right)\left|\phi_{p}\right| / \phi_{p} \in \operatorname{Belt}\left(\bar{\partial} V, R_{p}\right)$, we get from (4.3)-(4.5) that

$$
\lim _{q \rightarrow p} \operatorname{Re} \iint_{R} \frac{\left|\phi_{p}\right|}{\phi_{p}} \frac{z-z_{0}}{\left|z-z_{0}\right|} \phi_{p, q}=1
$$

which implies that $\left(\left(z-z_{0}\right) /\left|z-z_{0}\right| \phi_{p, q}\right)$ is a Hamilton sequence for the infinitesimal extremal Beltrami differential $\lambda\left(z_{0}\right)\left|\phi_{p}\right| / \phi_{p}$ as $q \rightarrow p$. Since $\lambda\left(z_{0}\right)\left|\phi_{p}\right| / \phi_{p}$ represents an infinitesimal Reich-Strebel point, $\left(\left(z-z_{0}\right) /\left|z-z_{0}\right| \phi_{p, q}\right)$ must converge in norm to $\phi_{p}$ as $q \rightarrow p$. This finishes the proof of the first conclusion.

Noting that $T_{q, p}=T_{p, q}^{-1}$, we conclude that

$$
\phi_{p, q}=-\left(1-k_{p, q}^{2}\right) \phi_{q, p} \circ T_{p, q}\left(\partial T_{p, q}\right)^{2} .
$$

So the second conclusion follows from the first one.

Example 1. When $R=\Delta, \pi=i d$, and the computation in Section 3 shows that

$$
\phi_{z_{0}}=-\frac{1}{2 \pi} \frac{\left(1-\left|z_{0}\right|^{2}\right)^{2}}{\left(1-\overline{z_{0}} \zeta\right)^{3}\left(\zeta-z_{0}\right)} .
$$

So in this case we have

$$
\lim _{z \rightarrow z_{0}}\left\|\frac{z-z_{0}}{\left|z-z_{0}\right|} \phi_{z_{0}, z}+\frac{1}{2 \pi} \frac{\left(1-\left|z_{0}\right|^{2}\right)^{2}}{\left(1-\overline{z_{0}} \zeta\right)^{3}\left(\zeta-z_{0}\right)}\right\|=0 .
$$

Particularly, for the classical Teichmüller shift mapping, $\left\|\phi_{\delta}-1 / 2 \pi \zeta\right\| \rightarrow 0$ as $\delta \rightarrow 0+$. On the other hand, by (4.2) we obtain that for any $z_{0} \in \Delta$ and any $\phi \in A\left(\Delta_{z_{0}}\right)$, it holds that

$$
\frac{1}{2 \pi\left(1-\left|z_{0}\right|^{2}\right)} \iint_{\Delta} \frac{\left(1-\overline{z_{0}} \zeta\right)^{3}\left(\zeta-z_{0}\right)}{\left|\left(1-\overline{z_{0}} \zeta\right)^{3}\left(\zeta-z_{0}\right)\right|} \phi=\operatorname{Res}_{\zeta=z_{0}} \phi .
$$

So we have the following reproducing formula for functions $\phi \in A(\Delta)$,

$$
\phi(z)=\frac{1}{2 \pi\left(1-|z|^{2}\right)} \iint_{\Delta} \frac{(1-\bar{z} \zeta)^{3}}{\left|(1-\bar{z} \zeta)^{3}(\zeta-z)\right|} \phi .
$$

\section{Smoothness of the distance $d_{K}$ and the Teichmüller density $\lambda$}

We choose $p_{0} \in R$ and consider the map $F: R \rightarrow T\left(R_{p_{0}}\right)$ defined as

$$
F(p)=\Phi\left(\mu_{p_{0}, p}\right),
$$

where $\mu_{p_{0}, p}$ is the Beltrami coefficient of the Teichmüller shift mapping $T_{p_{0}, p}$. By a result of Bers [Be] (see also [Na2]), $F$ is a holomorphic mapping. By definition,

$$
d_{K}\left(p_{1}, p_{2}\right)=d_{T}\left(F\left(p_{1}\right), F\left(p_{2}\right)\right),
$$

so $G_{K}=F^{*} G_{T}$, or more precisely, $G_{K}\left(p, v_{p}\right)=G_{T}\left(F(p), d_{p} F\left(v_{p}\right)\right)$, that is, the infinitesimal form $G_{K}$ is the pull-back of the Teichmüller metric $G_{T}$ under the 
holomorphic mapping $F$. On the other hand, it is well known that $G_{T}$ is continuous on the whole tangent bundle, and it is even continuously differentiable except at the points on the zero section when $R$ is of conformally finite type (see $[\mathrm{Ga}]$ ). Since the map $F$ is holomorphic, we obtain the following theorem.

Theorem 4. The infinitesimal form $G_{K}$ is continuous on the whole bundle TR. Consequently, the Teichmüller density $\lambda$ is continuous on the whole surface $R$. Furthermore, when $R$ is of conformally finite type, $G_{K}$ is continuously differentiable except at the points on the zero section, so $\lambda$ is continuously differentiable on the whole surface $R$.

Remark 5. It is known that the holomorphic curvature of the Teichmüller metric $G_{T}$ is identically equal to -4 (see $[\mathrm{AP}]$ for more details). So the holomorphic curvature of $G_{K}$ is bounded above by -4 .

Remark 6. Let $d_{K}^{i}$ denote the inner distance induced by $d_{K}$, or equivalently, it is the integral of the infinitesimal form $G_{K}$. Since $F(R) \subset T\left(R_{p_{0}}\right)$ does not contain any Teichmüller geodesic segment (see [EL2], Theorem 2), we conclude that $d_{K}<d_{K}^{i}$ on the whole surface unless $R=\overline{\mathbb{C}}_{p q r}$, so $d_{K}$ is not an inner distance. It is known that both the hyperbolic distance $d_{H}$ and the Teichmüller distance $d_{T}$ are inner.

An immediate consequence of Theorem 4 is the continuity of extremal differentials. For any point $p \in R$, under the local parameter $z=\pi^{-1}$, $\lambda(z)=-\pi \operatorname{Res}_{\zeta=z} \phi_{p}$. Now let $p_{n} \rightarrow p$. By the continuity of $\lambda, \operatorname{Res}_{\zeta=z_{n}} \phi_{p_{n}} \rightarrow$ $\operatorname{Res}_{\zeta=z} \phi_{p}$. On the other hand, since $\left(\phi_{p_{n}}\right)$ is a normal family, we conclude without loss of generality that $\left(\phi_{p_{n}}\right)$ converges to some $\phi \in A\left(R_{p}\right)$ locally uniformly in $R_{p}$. By Fatou's Lemma, $\|\phi\| \leq 1$. Since $\operatorname{Res}_{\zeta=z_{n}} \phi_{p_{n}} \rightarrow \operatorname{Res}_{\zeta=z} \phi$, $\operatorname{Res}_{\zeta=z} \phi=\operatorname{Res}_{\zeta=z} \phi_{p}$, so we must have $\|\phi\|=1$ and so $\phi=\phi_{p}$. Consequently, by Lebesgue's dominated convergence theorem we have $\left\|\phi_{p_{n}}-\phi_{p}\right\| \rightarrow 0$. We have proved

Theorem 5. $\quad \lim _{q \rightarrow p}\left\|\phi_{q}-\phi_{p}\right\|=0$.

We also have some corresponding properties of the distance function $d_{K}$. We consider the continuous function $G: R \rightarrow \mathbb{R}$ defined as

$$
G(p)=d_{K}\left(p_{0}, p\right)=d_{T}\left(F\left(p_{0}\right), F(p)\right)=d_{T}\left(\Phi(0), \Phi\left(\mu_{p_{0}, p}\right) .\right.
$$

When $R$ is of conformally finite type, it is known that $d_{T}$ is continuously differentiable except at the points on the diagonal (see $[\mathrm{Ea}]$ or $[\mathrm{Ga}]$ ). Since $F$ is holomorphic, $G$ is continuously differentiable whenever $p \neq p_{0}$. When $R$ is of conformally infinite type, by a result of Lakic [La], the function $\Phi(\nu) \rightarrow$ $d_{T}(\Phi(0), \Phi(\nu))$ is continuously differentiable at $\Phi(\mu)$ if it is a Strebel point. Clearly, when $p \neq p_{0}, F(p)$ is a Strebel point in $T\left(R_{p_{0}}\right)$. So $G$ is again continuously differentiable whenever $p \neq p_{0}$. So we have

Theorem 6. The function $G: R \rightarrow \mathbb{R}$ is continuously differentiable except at the point $p_{0}$. Consequently, the distance function $d_{K}: R \times R \rightarrow \mathbb{R}$ is continuously differentiable off the diagonal. 
Remark 7. Recall that

$$
k_{p_{0}, p}=\frac{e^{2 d_{K}\left(p_{0}, p\right)}-1}{e^{2 d_{K}\left(p_{0}, p\right)}+1}=\frac{e^{2 G(p)}-1}{e^{2 G(p)}+1},
$$

so $k_{p_{0}, p}$ is also continuously differentiable except at the point $p_{0}$. It is also logarithmically pluri-subharmonic on the whole surface $R$. In fact, let $g_{T}$ denote the Green function of a Teichmüller space, then (see [Kru2])

$$
g_{T}=\log \frac{e^{2 d_{T}}-1}{e^{2 d_{T}}+1}
$$

and so

$$
g_{T}\left(F\left(p_{0}\right), F(p)\right)=\log \frac{e^{2 d_{K}\left(p_{0}, p\right)}-1}{e^{2 d_{K}\left(p_{0}, p\right)}+1}=\log \frac{e^{2 G(p)}-1}{e^{2 G(p)}+1}=\log k_{p_{0}, p},
$$

which implies that $k_{p_{0}, p}$ is logarithmically pluri-subharmonic on the whole surface $R$.

In the rest of the section, we shall give the derivatives of the mapping $F: R \rightarrow T\left(R_{p_{0}}\right)$ and the function $G: R \rightarrow \mathbb{R}$, which is of independent interest.

Proposition 7. Under the local parameter $z=\pi^{-1}$, for any $z \in \Delta$ and non-zero $v \in \mathbb{C}$,

$$
\begin{aligned}
& d_{z}(F \circ \pi)(v) \\
& \quad=d_{\mu_{p_{0}, \pi(z)}} \Phi\left(\left(1-k_{p_{0}, \pi(z)}^{2}\right) G_{K}\left(\pi(z), d_{z} \pi(v)\right) \frac{v}{|v|} \frac{\left|\phi_{\pi(z)}\right|}{\phi_{\pi(z)}} \circ T_{p_{0}, \pi(z)} \frac{\overline{\partial T_{p_{0}, \pi(z)}}}{\partial T_{p_{0}, \pi(z)}}\right) .
\end{aligned}
$$

Proof. Since we have already known that $F$ is holomorphic, we only need to calculate

$$
\begin{aligned}
& d_{z}(F \circ \pi)(v) \\
& \quad=\lim _{t \rightarrow 0+} \frac{F(\pi(z+t v))-F(\pi(z))}{t}=\lim _{t \rightarrow 0+} \frac{\Phi\left(\mu_{p_{0}, \pi(z+t v)}\right)-\Phi\left(\mu_{p_{0}, \pi(z)}\right)}{t} .
\end{aligned}
$$

Let $\mu_{t}$ denote the Beltrami coefficient of the mapping $T_{\pi(z), \pi(z+t v)} \circ T_{p_{0}, \pi(z)}$, then $\Phi\left(\mu_{t}\right)=\Phi\left(\mu_{p_{0}, \pi(z+t v)}\right)=F(\pi(z+t v))$ and it is a holomorphic mapping. Now

$$
\mu_{t}=\frac{\mu_{p_{0}, \pi(z)}+\mu_{\pi(z), \pi(z+t v)} \circ T_{p_{0}, \pi(z)} \frac{\overline{\partial T_{p_{0}, \pi(z)}}}{\partial T_{p_{0}, \pi(z)}}}{1+\bar{\mu}_{p_{0}, \pi(z)} \mu_{\pi(z), \pi(z+t v)} \circ T_{p_{0}, \pi(z)} \frac{\overline{\partial T_{p_{0}, \pi(z)}}}{\partial T_{p_{0}, \pi(z)}}} .
$$

Since

$$
\begin{aligned}
& \mu_{\pi(z), \pi(z+t v)} \\
& \quad=k_{\pi(z), \pi(z+t v)} \frac{\left|\phi_{\pi(z), \pi(z+t v)}\right|}{\phi_{\pi(z), \pi(z+t v)}}=\left(t G_{K}\left(\pi(z), d_{z} \pi(v)\right)+o(t)\right) \frac{\left|\phi_{\pi(z), \pi(z+t v)}\right|}{\phi_{\pi(z), \pi(z+t v)}},
\end{aligned}
$$


and by Theorem $3,\left\|v \phi_{\pi(z), \pi(z+t v)}-|v| \phi_{\pi(z)}\right\| \rightarrow 0$ as $t \rightarrow 0+$, we conclude by (5.7) that

$$
\mu^{\prime}(0+)=\left(1-k_{p_{0}, \pi(z)}^{2}\right) G_{K}\left(\pi(z), d_{z} \pi(v)\right) \frac{v}{|v|} \frac{\left|\phi_{\pi(z)}\right|}{\phi_{\pi(z)}} \circ T_{p_{0}, \pi(z)} \frac{\overline{\partial T_{p_{0}, \pi(z)}}}{\partial T_{p_{0}, \pi(z)}} .
$$

Consequently, by (5.6) and (5.8) we obtain as required that

$$
\begin{aligned}
& d_{z}(F \circ \pi)(v)=\left.\frac{d}{d t} \Phi\left(\mu_{t}\right)\right|_{t=0+}=d_{\mu_{0}} \Phi\left(\mu^{\prime}(0+)\right) \\
& \quad=d_{\mu_{p_{0}, \pi(z)}} \Phi\left(\left(1-k_{p_{0}, \pi(z)}^{2}\right) G_{K}\left(\pi(z), d_{z} \pi(v)\right) \frac{v}{|v|} \frac{\left|\phi_{\pi(z)}\right|}{\phi_{\pi(z)}} \circ T_{p_{0}, \pi(z)} \frac{\overline{\partial T_{p_{0}, \pi(z)}}}{\partial T_{p_{0}, \pi(z)}}\right)
\end{aligned}
$$

Using the formula of $d F$, we can examine directly that $G_{K}=F^{*} G_{T}$. In fact, under the local parameter $z=\pi^{-1}$, by $(2.19)$,

$$
\begin{aligned}
G_{T} & \left(F(\pi(z)), d_{z}(F \circ \pi)(v)\right) \\
& =G_{T}\left(\Phi\left(\mu_{p_{0}, \pi(z)}\right), d_{\mu_{p_{0}, \pi(z)}} \Phi\left(\mu^{\prime}(0+)\right)\right) \\
& =\sup _{\phi \in S A\left(R_{\pi(z)}\right)}\left|\iint_{R}\left(\frac{\mu^{\prime}(0+)}{1-k_{p_{0}, \pi(z)}^{2}} \frac{\partial T_{p_{0}, \pi(z)}}{\partial T_{p_{0}, \pi(z)}}\right) \circ\left(T_{p_{0}, \pi(z)}\right)^{-1} \phi\right| \\
& =\sup _{\phi \in S A\left(R_{\pi(z)}\right)}\left|\iint_{R} G_{K}\left(\pi(z), d_{z} \pi(v)\right) \frac{v}{|v|} \frac{\left|\phi_{\pi(z)}\right|}{\phi_{\pi(z)}} \phi\right|=G_{K}\left(\pi(z), d_{z} \pi(v)\right) .
\end{aligned}
$$

Now we give the derivative of the function $G$. Noting that

$$
\begin{aligned}
G(\pi(z+t v)) & =d_{T}(\Phi(0), F(\pi(z+t v))) \\
& =d_{T}\left(\Phi(0), F(\pi(z))+t d_{z}(F \circ \pi)(v)+o(t)\right),
\end{aligned}
$$

when $z \in \Delta-\pi^{-1}\left(p_{0}\right), F(\pi(z))$ is a Strebel point in $T\left(R_{p_{0}}\right)$, by Lemma 2 in [La], we can obtain

$$
\begin{aligned}
d_{z}(G \circ \pi)(v) & =\frac{1}{1-k_{p_{0}, \pi(z)}^{2}} \operatorname{Re} \iint_{R} \mu^{\prime}(0+) \phi_{p_{0}, \pi(z)} \\
& =\operatorname{Re} \iint_{R} G_{K}\left(\pi(z), d_{z} \pi(v)\right) \frac{v}{|v|} \frac{\left|\phi_{\pi(z)}\right|}{\phi_{\pi(z)}} \circ T_{p_{0}, \pi(z)} \frac{\overline{\partial T_{p_{0}, \pi(z)}}}{\partial T_{p_{0}, \pi(z)}} \phi_{p_{0}, \pi(z)} \\
& =-\operatorname{Re} \iint_{R} G_{K}\left(\pi(z), d_{z} \pi(v)\right) \frac{v}{|v|} \frac{\left|\phi_{\pi(z)}\right|}{\phi_{\pi(z)}} \phi_{\pi(z), p_{0}} \\
& =\pi \operatorname{Re}\left(v \operatorname{Res}_{\zeta=z} \phi_{\pi(z), p_{0}}\right) .
\end{aligned}
$$


When $z \in \pi^{-1}\left(p_{0}\right)$, by Proposition D and (5.9) we obtain

$$
\begin{aligned}
G(\pi(z+t v)) & =d_{T}\left(\Phi(0), t d_{z}(F \circ \pi)(v)+o(t)\right) \\
& =|t| \sup _{\phi \in S A\left(R_{p_{0}}\right)}\left|\iint_{R} G_{K}\left(\pi(z), d_{z} \pi(v)\right) \frac{v}{|v|} \frac{\left|\phi_{\pi(z)}\right|}{\phi_{\pi(z)}} \phi\right|+o(t) \\
& =|t| G_{K}\left(\pi(z), d_{z} \pi(v)\right)+o(t)=-|t| \pi|v| \operatorname{Res}_{\zeta=z} \phi_{p_{0}}+o(t) \\
& =|t v| \lambda(z)+o(t) .
\end{aligned}
$$

Clearly, $G$ is not differentiable at $p_{0}$.

We state the above discussion as a proposition, which can be served as a supplement to Theorem 6 .

Proposition 8. The function $G$ is not differentiable at $p_{0}$. Under the local parameter $z=\pi^{-1}$, for any $z \in \Delta-\pi^{-1}\left(p_{0}\right)$ and non-zero $v \in \mathbb{C}$, it holds that

$$
\begin{aligned}
d_{z}(G \circ \pi)(v) & =-\operatorname{Re} \iint_{R} G_{K}\left(\pi(z), d_{z} \pi(v)\right) \frac{v}{|v|} \frac{\left|\phi_{\pi(z)}\right|}{\phi_{\pi(z)}} \phi_{\pi(z), p_{0}} \\
& =\pi \operatorname{Re}\left(v \operatorname{Res}_{\zeta=z} \phi_{\pi(z), p_{0}}\right) .
\end{aligned}
$$

There is a generalization of the concept of Teichmüller shift mapping, which is called a point shift mapping. More precisely, let $Q: S \rightarrow R$ be a quasiconformal mapping, and $q_{0} \in S$ is a fixed point. An extremal mapping $f_{Q, p}$ which sends $q_{0}$ to a point $p \in R$ and is homotopic to $Q$ modulo $\partial S$ is called a point shift mapping. Note that $f_{Q, p}$ need not be uniquely extremal. Let $E(Q)$ denote the set of extremal mappings in the class of quasiconformal mappings which are homotopic to $Q$ modulo $\partial S$ and set

$$
V_{Q}=\left\{p=f\left(q_{0}\right): f \in E(Q)\right\} .
$$

Then $V_{Q}$ is called the variablity set of $Q$ with respect to $q_{0}$ (see [St2], [EL1], [EL2]). Strebel [St2], Earle and Lakic [EL1-2] proved that $V_{Q}$ is compact, connected and simply connected. When $p \in R-V_{Q}, f_{Q, p}$ is uniquely extremal, it is a Teichmüller mapping with Beltrami coefficient $\mu_{Q, p}=k_{Q, p}\left|\psi_{Q, p}\right| / \psi_{Q, p}$, where $k_{Q, p}>0, \psi_{Q, p} \in S A\left(S_{q_{0}}\right)$ has a simple pole at $q_{0}$. The inverse mapping $f_{Q, p}^{-1}$ is also a Teichmüller mapping with Beltrami coefficient $k_{Q, p}\left|\phi_{Q, p}\right| / \phi_{Q, p}$, where $\phi_{Q, p} \in S A\left(R_{p}\right)$ has a simple pole at $p$ and $\psi_{Q, p}=-\left(1-k_{Q, p}^{2}\right) \phi_{Q, p}$ 。 $f_{Q, p}\left(\partial f_{Q, p}\right)^{2}$.

Consider $F_{Q}: R \rightarrow T\left(S_{q_{0}}\right)$ and $G_{Q}: R \rightarrow \mathbb{R}$ as

$$
\begin{aligned}
& F_{Q}(p)=\Phi\left(\mu_{Q, p}\right), \\
& G_{Q}(p)=d_{T}\left(\Phi(0), F_{Q}(p)\right)=d_{T}\left(\Phi(0), \Phi\left(\mu_{Q, p}\right)\right) .
\end{aligned}
$$

Then $F_{Q}$ is holomorphic on $R$ and $G_{Q}$ is continuously differentiable in $R-V_{Q}$. Note that we still have $d_{K}\left(p_{1}, p_{2}\right)=d_{T}\left(F_{Q}\left(p_{1}\right), F_{Q}\left(p_{2}\right)\right)$ and so $G_{K}=$ $F_{Q}^{*} G_{T}$. By the same reasoning as above, we can obtain 
Proposition 9. Under the local parameter $z=\pi^{-1}$, for any $z \in \Delta$ and non-zero $v \in \mathbb{C}$,

$$
\begin{aligned}
& d_{z}\left(F_{Q} \circ \pi\right)(v) \\
& \quad=d_{\mu_{Q, \pi(z)}} \Phi\left(G_{K}\left(\pi(z), d_{z} \pi(v)\right) \frac{v}{|v|}\left(1-\left|\mu_{Q, \pi(z)}\right|^{2}\right) \frac{\left|\phi_{\pi(z)}\right|}{\phi_{\pi(z)}} \circ f_{Q, \pi(z)} \frac{\overline{\partial f_{Q, \pi(z)}}}{\partial f_{Q, \pi(z)}}\right) .
\end{aligned}
$$

Proposition 10. Under the local parameter $z=\pi^{-1}$, for any $z \in \Delta-$ $\pi^{-1}\left(V_{Q}\right)$ and non-zero $v \in \mathbb{C}$, it holds that

$$
\begin{aligned}
d_{z}\left(G_{Q} \circ \pi\right)(v) & =-\operatorname{Re} \iint_{R} G_{K}\left(\pi(z), d_{z} \pi(v)\right) \frac{v}{|v|} \frac{\left|\phi_{\pi(z)}\right|}{\phi_{\pi(z)}} \phi_{Q, \pi(z)} \\
& =\pi \operatorname{Re}\left(v \operatorname{Res}_{\zeta=z} \phi_{Q, \pi(z)}\right) .
\end{aligned}
$$

For further properties of the function $G_{Q}$, see Theorem 3 in [EL2].

Acknowledgements. The author would like to thank Nikola Lakic for sending to him a series of papers, which were greatly helpful for him. The author also thanks the referee for his (her) valuable suggestions, which make the paper be more readable.

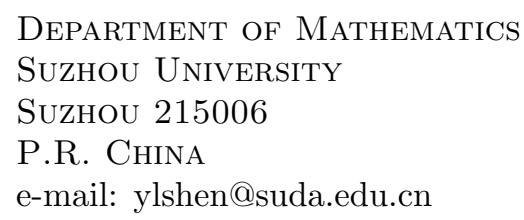

\section{References}

[AP] M. Abate and G. Patrizio, Isometries of the Teichmüller metric, Ann. Scuola Norm. Sup. Pisa CL. Sci. XXVI (1998), 437-452.

[Be] L. Bers, Fiber spaces over Teichmüller spaces, Acta Math. 130 (1973), 89-126.

[Ea] C. J. Earle, The Teichmüller distance is differentiable, Duke Math. J. 44 (1977), 389-397.

[EG] C. J. Earle and F. P. Gardiner, Geometric isomorphisms between infinite dimensional Teichmüller spaces, Trans. Amer. Math. Soc. 348 (1996), 1163-1190.

[EKK] C. J. Earle, I. Kra and S. L. Krushkal, Holomorphic motions and Teichmüller spaces, Trans. Amer. Math. Soc. 343 (1994), 927-948.

[EL1] C. J. Earle and N. Lakic, Variablity set on a Riemann surface, preprint. 
[EL2] C. J. Earle and N. Lakic, Variablity sets on Riemann surfaces and forgetful maps between Teichmüller spaces, Ann. Acad. Sci. Fenn. A I Math. 27 (2002), 307-324.

[ELi] C. J. Earle and Z. Li, Isometrically embedded polydisks in infinite dimensional Teichmüller spaces, J. Geom. Anal. 9 (1999), 51-71.

[Ga] F. P. Gardiner, Teichmüller Theory and Quadratic Differentials, WileyInterscience, New York, 1987.

[GL1] F. P. Gardiner and N. Lakic, Quasiconformal Teichmüller Theory, AMS, Providence, R. I., 2000.

[GL2] _ Comparing Poincaré densities, Ann. of Math. 154 (2001), 245267.

[Ge] F. G. Gehring, Quasiconformal mappings which hold the real axis pointwise fixed, Ohio Univ. Press, Athens, Ohio, 1970, pp. 145-148.

[Ha] R. S. Hamilton, Extremal quasiconformal mappings with prescribed boundary values, Trans. Amer. Math. Soc. 138 (1969), 399-406.

[Kr] I. Kra, On the Nielsen-Thurston-Bers type of some self-maps of Riemann surfaces, Acta Math. 146 (1981), 231-270.

[Kru1] S. L. Krushkal, Extremal quasiconformal mappings, Siberian Math. J. 10 (1969), 411-418.

[Kru2] _ The Green function of Teichmüller spaces with applications, Bull. Amer. Math. Soc. 27 (1992), 143-147.

[Kru3] - Univalent functions and holomorphic motions, J. Anal. Math. 66 (1995), 252-275.

[La] N. Lakic, Strebel points, Proceedings of the Bers' Colloquium, AMS 1997.

[Li] Z. Li, Quasiconformal Mappings and Their Applications to the Theory of Riemann Surfaces (Chinese), Chinese Academic Press, 1988.

[Liu] L. Liu, Invariant metrics in infinite dimensional Teichmüller spaces, Complex Variables 25 (1994), 337-349.

[Na1] S. Nag, Nongedosic disks embedded in Teichmüller spaces, Amer. J. Math. 104 (1982), 399-408.

[Na2] , The Complex Analytic Theory of Teichmüller Spaces, WileyInterscience, 1988.

[Re] E. Reich, On the mappings with complex dilatation $k e^{i \theta}$, Ann. Acad. Sci. Fenn. A I Math. 12 (1987), 261-267. 
[RS1] E. Reich and K. Strebel, On quasiconformal mappings which keep the boundary points fixed, Trans. Amer. Math. Soc. 137-138 (1969), 211221.

[RS2] - Extremal quasiconformal mappings with prescribed boundary values, In: Contributions to Analysis, A collection of papers dedicated to Lipman Bers, Academic Press, New York, 1974, pp. 375-391.

[St1] K. Strebel, Extremal quasiconformal mappings, Result. Math. 10 (1986), 169-209.

[St2] , Point-shift differentials and extremal quasiconforml mappings, Ann. Acad. Sci. Fenn. A I Math. 23 (1998), 475-494.

[Te] O. Teichmüller, Ein Verschiebungssatz der quasikonformem Abbildung, Deutsche Mathematik (1944), 336-343. 